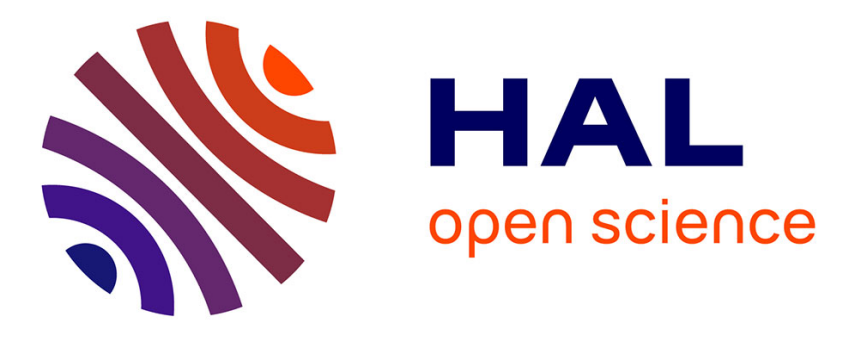

\title{
On the stock estimation for some fishery systems
}

Aboudramane Guiro, Abderrahman Iggidr, Diène Ngom, Hamidou Touré

\section{To cite this version:}

Aboudramane Guiro, Abderrahman Iggidr, Diène Ngom, Hamidou Touré. On the stock estimation for some fishery systems. Reviews in Fish Biology and Fisheries, 2009, 19 (3), pp.313-327. 10.1007/s11160-009-9104-7 . inria-00595295

\section{HAL Id: inria-00595295 \\ https://hal.inria.fr/inria-00595295}

Submitted on 24 May 2011

HAL is a multi-disciplinary open access archive for the deposit and dissemination of scientific research documents, whether they are published or not. The documents may come from teaching and research institutions in France or abroad, or from public or private research centers.
L'archive ouverte pluridisciplinaire HAL, est destinée au dépôt et à la diffusion de documents scientifiques de niveau recherche, publiés ou non, émanant des établissements d'enseignement et de recherche français ou étrangers, des laboratoires publics ou privés. 


\title{
On the stock estimation for some fishery systems
}

\author{
A. Guiro ${ }^{a, b}$, A. Iggidr ${ }^{b *}$ D. Ngom ${ }^{b, c}$, and H. Touré ${ }^{a}$ \\ ${ }^{a}$ Laboratoire d'Analyse Mathématique des Equations (LAME) \\ Faculté des Sciences et Techniques \\ Université de Ouagadougou Bp: 7021 Ouagadougou, Burkina Faso. \\ ${ }^{b}$ INRIA Nancy - Grand Est and University Paul Verlaine-Metz \\ LMAM-CNRS UMR 7122 \\ ISGMP Bat. A, Ile du Saulcy 57045 Metz Cedex 01, France. \\ ${ }^{c}$ Laboratoire d'Analyse Numérique et d'Informatique (LANI) \\ UFR de Sciences Appliquées et de Technologie \\ Université Gaston Berger. B.P. 234 Saint-Louis, Sénégal.
}

\begin{abstract}
In this work we address the stock estimation problem for two fishery models. We show that a tool from nonlinear control theory called "observer" can be helpful to deal with the resource stock estimation in the field of renewable resource management. It is often difficult or expensive to measure all the state variables characterising the evolution of a given population system, therefore the question arises whether from the observation of certain indicators of the considered system, the whole state of the population system can be recovered or at least estimated. The goal of this paper is to show how some techniques of control theory can be applied for the approximate estimation of the unmeasurable state variables using only the observed data together with the dynamical model describing the evolution of the system. More precisely we shall consider two fishery models and we shall show how to built for each model an auxiliary dynamical system (the observer) that uses the available data (the total of caught fish) and which produces a dynamical estimation $\hat{x}(t)$ of the unmeasurable stock state $x(t)$. Moreover the convergence speed of $\hat{x}(t)$ towards $x(t)$ can be chosen.
\end{abstract}

Keywords: Fishery models, Stage-structured population models, Estimation, Harvested Fish Population, Observers.

\footnotetext{
${ }^{*}$ Corresponding author: e-mail: iggidr@loria.fr, iggidr@math.univ-metz.fr
} 


\section{Introduction and a short survey of observers de- sign}

3 The stock estimation is one of the most important problem in fishery science. One can quote J.A. Gulland [17]: A major emphasis in fishery science has been on the problems of estimating current and past level using catch levels and fishing effort data.

To make a policy decision about the exploitation of renewable ressources, it is necessary to take into account the state of the resource stocks. This implies the need of a good estimate of the available resource. Mathematical models are more and more used to describe the evolution of biological systems. Here, we consider two mathematical models for fishery resources. The first one is a "stage structured" model $[43,44]$ that describes the dynamics of a population divided in stage-classes (according to age, length or weight) and submitted to the fishing action. The second model is a "global" model that describes the evolution of a fish population that can move between an area where it can be harvested and a reserve area where no fishing is allowed [9]. Both models are given by systems of differential equations of the form

$$
\dot{x}=f(x, E),
$$

where $E$ is the fishing effort (it can be seen as a control or an input) and $x(t)$ is the state of the system at time $t$. The state variable $x(t)$ represents the density of the population or the number of individuals by stage. For both models, the state $x(t)$ is not available for measurement. In practice, the only available information at time $t$ is the value of the captures: this means that one can measure the total catch at each time $t$. The value of the captures can be seen as the measurable output of system (1). The output is in general a function of the state variable and the input, that is, $y(t)=h(x(t), E)$.

Now assuming that (1) is a "good" model of the system under consideration, if it is possible to have the value of the state at some time $t_{0}$ then it is possible to compute $x(t)$ for all $t \geq t_{0}$ by integrating the differential equation with the initial condition $x\left(t_{0}\right)$. Unfortunately, it is often not possible to measure the whole state at a given time and therefore it is not possible to integrate the differential equation because one does not know an initial condition. One can only have a partial information of the state and this partial information is precisely given by $y(t)$ the output of the system. Therefore we shall show how to use this partial information $y(t)$ together with the given model in order to have a dynamical estimate $\hat{x}(t)$ of the real unknown state variable $x(t)$. This estimate will be produced by an auxiliary dynamical system which uses the information $y(t)$ provided by the system (1). This dynamical system is generally of the form

$$
\dot{\hat{x}}=g(\hat{x}, E, y) \text {. }
$$

It can be represented by Schema 1 The estimate error is given by $e(t)=\hat{x}(t)-x(t)$ and it satisfies the following "error equation"

$$
\dot{e}=g(\hat{x}, E, y)-f(x, E)
$$




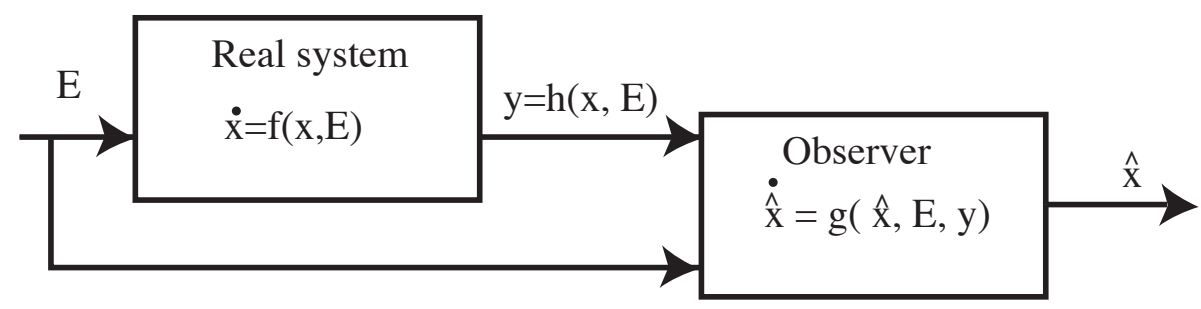

Figure 1: A schematic representation of an observer

39 The function $g$ has to be determined in such a way that the solutions of (1) and (2) 40 satisfy $x(t)-\hat{x}(t) \rightarrow 0$ as $t \rightarrow+\infty$ regardless of the respective initial conditions of 41 system (1) and system (2).

42 A dynamical system (2) satisfying this conditions is called an "observer" for system (1). When the convergence of $\hat{x}(t)$ towards $x(t)$ is exponential, the system (2) is an "exponential observer". More precisely, system (2) is an exponential observer for system (1) if there exists $\lambda>0$ such that, for all $t \geq 0$ and for all initial conditions $(x(0), \hat{x}(0))$, the corresponding solutions of (1) and (2) satisfy

$$
\|\hat{x}(t)-x(t)\| \leq \exp (-\lambda t)\|\hat{x}(0)-x(0)\| .
$$

In this situation a good estimate of the real unmeasured state is rapidly obtained. One must notice that we need not care about the choice of the initial condition of the observer since the convergence of $\hat{x}(t)$ towards the real state $x(t)$ does not depend on this choice.

When the system under consideration is a linear system, i.e., it can be written as follows

$$
\left\{\begin{array}{l}
\dot{x}(t)=A x(t)+B u(t), \\
y(t)=C x(t), \\
x(t) \in \mathbb{R}^{n}, u(t) \in U \subset \mathbb{R}^{m}, y(t) \in \mathbb{R}^{q}, \\
A, B, \text { and } C \text { are respectively } n \times n, n \times m \text { and } q \times n \text { matrices, }
\end{array}\right.
$$

then an exponential observer (called Luenberger Observer)[30] for this system is given by

$$
\dot{\hat{x}}(t)=A \hat{x}(t)+B u(t)+K(y(t)-C \hat{x}(t))
$$

where the $n \times q$ matrix $K$ has to be computed. The Luenberger observer converges, i.e., $|\hat{x}(k)-x(k)|$ tends to zero exponentially fast if it is possible to find a matrix $K$ in such a way that the eigenvalues of the matrix $A-K C$ are all with negative real part. It has been proved that such a matrix $K$ exists if the pair $(C, A)$ is observable. 
The pair $(C, A)$ is observable if and only if the matrix:

$$
O_{(C, A)}=\left(\begin{array}{c}
C \\
C A \\
C A^{2} \\
\vdots \\
C A^{n-1}
\end{array}\right)
$$

is of rank $n$. In this case we say that the system (4), or the pair $(C, A)$, satisfies the Kalman rank condition for observability (one can see for more details and examples [39] or [20]).

The construction of observers for highly nonlinear systems is still a very active research area in Control Theory. Several methods have been developed for some classes of systems (one can see for instance the references [30, 27, 28, 26, 47, 13] that represent different approaches). This is not an exhaustive list, because the literature on the subject is very extensive. This active research has resulted in the emergence of many nonlinear observer design techniques. The most classical one is based on the "feedback linearization" and the observer normal form (see for instance [6], [22], [27], [46]) Roughly speaking, this method consists in finding change of coordinates $x=\kappa(z), u=\zeta(E), y=\eta(w)$ in the state space as well as in the input space and in the output space in such a way that equation (1) is transformed into

$$
\left\{\begin{array}{l}
\dot{x}=A x+\chi(w, u), \\
w=C x .
\end{array}\right.
$$

In this case a Luenberger type observer can be easily constructed. However the conditions under which the appropriate changes of coordinates exist are restrictive. These changes of coordinates often exist only locally and hence the derived observer design works only locally.

The second famous method is the high gain construction ([41],[7],[13],[14], [15], [21], [4]). A short survey is given in [4]. This method is developed hereafter and will be used in this paper.

Another design method uses an on-line optimization approach ([24] , [2], [33], [34], [47]) such as moving horizon observers that use the integral output prediction error in the estimation process, and the observer using Newtons method. In this case, the state is estimated by minimizing a certain norm of the difference between the ob- server output and the measured output. The advantage of the online optimization method is the capability of dealing with a variety of nonlinear systems including time-varying systems, chaotic systems, and systems with unknown parameters. Moreover this method does not require the use of any canonical form. However, the corresponding observer computations are generally quite heavy and may prevent the use of these observers for systems with very fast dynamics.

Historically observability theory and observers design have been developed for artificial engineering systems but nowadays they are more and more applied to "natural 
systems". We outline here some applications of nonlinear observers to biological models. Once again the list is not exhaustive.

In [5] the well-known Droop model which describes the growth of a population of phytoplanktonic cells is considered. Observers for this model are built and are used to discuss the validity of this model by comparing the prediction of the state computed by the observer with direct measurements of this state.

In [10], observers are used to estimate the kinetic rates in bioreactors. The efficiency of the observer design is illustrated with examples dealing with the microbial growth and biosynthesis reactions.

A robust nonlinear asymptotic observer with adjustable convergence rate has been proposed in[1]. This observer has been applied to a model of an anaerobic digestion process used for wastewater treatment.

The authors of [29] consider a system of populations described by the classical LotkaVolterra model with one predator and two preys. The only available information is the total quantity of population preys without distinction between them. An observer is constructed that allows to estimate all the state variables. It is also shown how the observer can be used for the estimation of the level of an abiotic effect on the population system. It must be, however, noticed that the proposed observer in [29] is a local observer, i.e., its convergence is guaranteed only if the initial estimate error is small.

A high gain observer is used in [42] to study a system describing a one-gene regulation circuit. The observer is used to to rebuild the non-measured concentrations of the mRNA and the protein.

The use of observer theory in fishery is scarce, we have done some works in this sense (see [35], [16]). In [35], an observer has been constructed for a stage structured discrete-time fishery model that exhibits an unknown recruitment function. In [16], a stage structured continuous model is considered and it is assumed that only the last class (mature individuals) is harvested. The present work is a continuation and a generalization of [16].

The goal of this paper is twofold. First we shall show that some tools from control theory are helpful to address the stock estimation problem for an exploited fish population. More precisely we shall built exponential observers for the two models under consideration. These observers will allow to give an estimate of the respective stocks. The second is to show that the application of mathematical tools to biological systems has to be done carefully. One of the most efficient way to build an observer for a nonlinear system has been given in [13]. We briefly recall the method developed in [13]. To simplify matters we consider systems without control. Roughly speaking, the result of [13] concerns systems that can be written (possibly after a coordinates 
change):

$$
\left\{\begin{array}{c}
\dot{z}(t)=\underbrace{\left(\begin{array}{ccccc}
0 & 1 & 0 & \ldots & 0 \\
0 & 0 & 1 & \ldots & 0 \\
\cdot & \cdot & . & . & \cdot \\
0 & 0 & \ldots & 0 & 1 \\
0 & 0 & \ldots & 0 & 0
\end{array}\right)}_{A} z(t)+\left(\begin{array}{c}
0 \\
0 \\
\vdots \\
0 \\
\psi(z(t))
\end{array}\right) \\
y(t)=z_{C}^{(1,0, \ldots, O)} z(t) .
\end{array}\right.
$$

The state of the system at time $t$ is $z(t)=\left(z_{1}(t), z_{2}(t), \ldots, z_{n}(t)\right) \in \mathbb{R}^{n}$, and its measurable output is $y(t)$. The fact that $y(t)=z_{1}(t)$ means that one can measure only the first component of the state and hence the other components are not available for measurement. Assume that the function $\psi$ is globally Lipschitz on $\mathbb{R}^{n}$, that is, there exists $K>0$ such that $|\psi(z)-\psi(x)| \leq K|z-x|$ for all $(z, x) \in \mathbb{R}^{n} \times \mathbb{R}^{n}$. It has then been proved in [13] that for $\theta \geq 1$ large enough, an exponential observer (a Luenberger type observer) for the system (7) is given by the following dynamical system:

$$
\dot{\hat{z}}=X(\hat{z})-S_{\theta}^{-1} C^{T}(C \hat{z}-y),
$$

with $S_{\theta}$ being the solution of

$$
\theta S_{\theta}+A^{T} S_{\theta}+S_{\theta} A=C^{T} C
$$

System (8) is an exponential observer for system (7) means that the solutions of (8) converge to the solutions of system (7) with an exponential speed regardless the values of the respective initial conditions $z(0)$ and $\hat{z}(0)$. To prove this result the authors of [13] use the fact that the function $\psi$ is globally Lipschitz on the whole state space $\mathbb{R}^{n}$. The global Lipschitz assumption is very restrictive. Biological systems always evolve in a bounded domain $\mathcal{D}$ of $\mathbb{R}^{n}$ and hence the global Lipschitz assumption is satisfied on $\mathcal{D}$. However, it must be noticed that the fact that the domain $\mathcal{D}$ is positively invariant for system (7) and that the map $\psi$ is globally Lipschitz on $\mathcal{D}$ does not guarantee the convergence of the observer (8) even if one take the initial values inside $\mathcal{D}$. Indeed, the domain $\mathcal{D}$ is positively invariant for the system (7) but it is not a positively invariant set for the system (8) defining the equations of the observer. More precisely, for a given initial condition $(z(0), \hat{z}(0)) \in$ $\mathcal{D} \times \mathcal{D}$, the corresponding solution $(z(t), \hat{z}(t))$ of (7-8) can leave the set $\mathcal{D} \times \mathcal{D}$ in finite time: the component $z(t)$ will actually belong to $\mathcal{D}$ for all positive time but there is no reason that the same property will be true for $\hat{z}(t)$. In order to built an exponential observer for the considered system in this situation, one has first to extend the function $\psi$ from $\mathcal{D}$ to the whole $\mathbb{R}^{n}$ by a function $\tilde{\psi}$ which is globally Lipschitz on $\mathbb{R}^{n}$ and then to consider the systems (7-8) defined on $\mathbb{R}^{n} \times \mathbb{R}^{n}$ after replacing the function $\psi$ by its prolongation $\tilde{\psi}$. The stage-structured fishery model we consider here will illustrate this fact. For this model, there is a domain $\mathcal{D} \subset \mathbb{R}^{3}$ which is positively invariant, and the system dynamics are defined by a vector field $X$ which is globally Lipschitz on $\mathcal{D}$. We shall show that the observer works well 
when we extend the vector field $X$ to the whole space $\mathbb{R}^{3}$ and it fails to work when the prolongation is not done. The same things are valid for the global model. This shows that the Lipschitz extension of the vector field mentioned in [13] is not only for mathematical sophistication purpose but it is also necessary for application purpose. Here we construct simply a continuous Lipschitz extension of the function $\psi$. For more details concerning the design of Lipschitz extensions one can see for instance [38].

The paper is organized as follows. In Section 2, we present the stage-structured model and we built an observer for this system. The construction is made for a three stages model. It can be done for an arbitrary number of stages but the calculus are longer and more complicated. Section 3 is devoted to the stock estimation problem for a "global" model. Once again, for clarity reasons, we have preferred to deal with a model with two fishing areas but the observer construction can be done for a system describing the dynamics of a fish population that can move between different fishing zones (an example of such a system has been considered in [32]).

\section{$2 \quad$ A Stage-structured model}

In this section, we consider a class of a structured model in fishery with three classes. The first class $x_{0}$ is constitute of the pre-recruits i.e the eggs, larvae and the juveniles. The second and the third classes are the post-recruits or the exploited phase of the population.

The dynamics of the system are modeled by the following three dimensional system (see [43, 44], [36]) :

$$
\left\{\begin{array}{l}
\dot{x}_{0}(t)=-\alpha_{0} x_{0}(t)+\sum_{i=1}^{2} f_{i} l_{i} x_{i}(t)-\sum_{i=1}^{2} p_{i} x_{i}(t) x_{0}(t)-p_{0} x_{0}^{2}(t) \\
\dot{x}_{1}(t)=\alpha x_{0}(t)-\left(\alpha_{1}+q_{1} E\right) x_{1}(t) \\
\dot{x}_{2}(t)=\alpha x_{1}(t)-\left(\alpha_{2}+q_{2} E\right) x_{2}(t)
\end{array}\right.
$$

where :

$x_{i}$ : the number of fish in the stage $i$.

$\alpha$ : linear aging coefficient

$m_{i}$ : natural mortality rate of class $i$

$\alpha_{i}=m_{i}+\alpha$

$p_{0}$ : juvenile competition parameter

$f_{i}$ : fecundity rate of class $i$

$l_{i}$ : reproduction efficiency of class $i$

$p_{i}$ : predation rate of class $i$ on class 0

$q_{i}$ : capturability coefficient of class $i$

$E$ : instantaneous fishing effort.

\author{
(in time $\mathrm{t}^{-1}$ ) \\ (in time $e^{-1}$ ) \\ (in time ${ }^{-1}$ ) \\ (in time ${ }^{-1}$.number ${ }^{-1}$ ) \\ (no dimension) \\ (in time ${ }^{-1}$ ) \\ $\left(\right.$ time $^{-1} \cdot$ num $^{-1}$ ) \\ (in unit effort ${ }^{-1}$ ) \\ (in unit effort $\times$ time $^{-1}$ ).
}

We assume that the total catch is available for measurement. This total catch can 
be considered as a measurable output of the system(9) and it is given by

$$
y(t)=q_{1} E x_{1}(t)+q_{2} E x_{2}(t)
$$

197

$$
{ }_{214} F(x(t))=\left(\begin{array}{c}
-\alpha_{0} x_{0}(t)+\sum_{i=1}^{2} f_{i} l_{i} x_{i}(t)-\sum_{i=1}^{2} p_{i} x_{i}(t) x_{0}(t)-p_{0} x_{0}^{2}(t) \\
\alpha x_{0}(t)-\left(\alpha_{1}+q_{1} E\right) x_{1}(t) \\
\alpha x_{1}(t)-\left(\alpha_{2}+q_{2} E\right) x_{2}(t)
\end{array}\right)
$$

215 Let $\Phi$ be the function $\Phi: \stackrel{o}{D} \rightarrow \mathbb{R}^{3}(\stackrel{o}{D}$ is the interior of $D)$, defined as follows:

We consider system (11) which is a nonlinear system. Our aim is to construct an observer (estimator) i.e an auxiliary system which will give a dynamical estimate $\left(\hat{x}_{0}(t), \hat{x}_{1}(t), \hat{x}_{2}(t)\right)$ of the state $\left(x_{0}(t), x_{1}(t), x_{2}(t)\right)$ of system $(9)$. For the construction of such auxiliary system, we shall use a method called High Gain construction (see for instance [13]). This construction provide an exponential observer; the estimation error will converges to zero with exponential speed, i.e.,

$$
\|\hat{x}(t)-x(t)\| \leq \exp (-\lambda t)\|\hat{x}(0)-x(0)\| .
$$

\subsection{High Gain observer design for (11)}

The system (11) is the system (9) coupled with the output (10). For the observer design, we will use the High Gain observer techniques (Gauthier et al.([13])) to construct a High Gain observer for system (9).

It has been proved in [43] that there is a positively invariant compact set for system (9). This set is of the form $D=\left[a_{0}, b_{0}\right] \times\left[a_{1}, b_{1}\right] \times\left[a_{2}, b_{2}\right]$, where the numbers $a_{i}$ can be chosen as small as we need and the numbers $b_{i}$ are function of the parameters $f_{i}, l_{i}$ and $p_{i}$. More precisely:

$$
\begin{aligned}
& b_{i}=\pi_{i} \mu \\
& \text { with } \pi_{i}=\frac{\alpha^{i}}{\prod_{j=1}^{i}\left(\alpha_{j}+q_{j} E\right)}, \\
& \text { and } \mu=\min _{i: p_{i} \neq 0}\left\{\frac{f_{i} l_{i}}{p_{i}}\right\}
\end{aligned}
$$

Let us denote by $F$ the vector field defining the dynamics of the system (9), and $h$ the output function, that is $y(t)=h(x(t))=q_{1} E x_{1}(t)+q_{2} E x_{2}(t)$ and 
${ }_{216} \Phi(x)=\left(\begin{array}{c}h(x) \\ L_{F} h(x) \\ L_{F}^{2} h(x)\end{array}\right)$, where $L$ denotes the Lie derivative operator with respect to 217 the vector field $F$. Thus,

$$
\Phi(x)=E\left(\begin{array}{c}
q_{1} x_{1}+q_{2} x_{2} \\
\alpha q_{1} x_{0}+\left(\alpha q_{2}-q_{1}\left(\alpha_{1}+q_{1} E\right)\right) x_{1}-q_{2}\left(\alpha_{2}+q_{2} E\right) x_{2} \\
\left(-\alpha_{0} \alpha q_{1}+\alpha^{2} q_{2}-\alpha q_{1}\left(\alpha_{1}+q_{1} E\right)\right) x_{0} \\
+\left(\alpha q_{1} f_{1} l_{1}-\alpha q_{2}\left(\alpha_{1}+q_{1} E\right)+q_{1}\left(\alpha_{1}+q_{1} E\right)^{2}-\alpha q_{2}\left(\alpha_{2}+q_{2} E\right)\right) x_{1} \\
+\left(\alpha q_{1} f_{2} l_{2}+q_{2}\left(\alpha_{2}+q_{2} E\right)^{2}\right) x_{2} \\
-\alpha q_{1} p_{0} x_{0}^{2}-\alpha q_{1} p_{1} x_{1} x_{0}-\alpha q_{1} p_{2} x_{2} x_{0}
\end{array}\right)
$$

${ }_{218}$ The Jacobian of $\Phi$ can be written:

$$
\frac{d \Phi}{d x}=\left(\begin{array}{ccc}
0 & q_{1} E & q_{2} E \\
\alpha q_{1} E & \gamma_{1} & \gamma_{2} \\
\gamma_{3} & \gamma_{4} & \gamma_{5}
\end{array}\right)
$$

and

$$
\left[\frac{d \Phi}{d x}\right]^{-1}=\frac{1}{\Gamma}\left(\begin{array}{ccc}
\beta_{1} & \beta_{2} & \beta_{3} \\
\beta_{4} & \beta_{5} & \beta_{6} \\
\beta_{7} & \beta_{8} & \beta_{9}
\end{array}\right)
$$

219 where:

$$
\begin{array}{ll}
{ }_{220} & \Gamma=\operatorname{Det}\left(\frac{d \Phi}{d x}\right)=q_{1} E \gamma_{2} \gamma_{3}+\alpha q_{1} q_{2} E^{2} \gamma_{4}-q_{2} E \gamma_{1} \gamma_{3}-\alpha q_{1}^{2} E^{2} \gamma_{5} \\
{ }_{221} & \gamma_{1}=\alpha q_{2} E-q_{1} E\left(\alpha_{1}+q_{1} E\right) \\
{ }^{222} & \gamma_{2}=-q_{2} E\left(\alpha_{2}+q_{2} E\right) \\
{ }_{223} & \gamma_{3}=\alpha^{2} q_{2} E-\alpha_{0} \alpha q_{1} E-\alpha q_{1} E\left(\alpha_{1}+q_{1} E\right)-2 \alpha q_{1} E p_{0} x_{0}-\alpha q_{1} E p_{1} x_{1}-\alpha q_{1} E p_{2} x_{2} \\
{ }_{224} & \gamma_{4}=q_{1} E\left(\alpha_{1}+q_{1} E\right)^{2}-\alpha q_{2} E\left(\alpha_{1}+q_{1} E\right)-\alpha q_{2} E\left(\alpha_{2}+q_{2} E\right)+\alpha q_{1} f_{1} l_{1} E-\alpha q_{1} E p_{1} x_{0} \\
{ }_{225} & \gamma_{5}=q_{2} E\left(\alpha_{2}+q_{2} E\right)^{2}+\alpha q_{1} f_{2} l_{2} E-\alpha q_{1} E p_{2} x_{0} \\
{ }_{226} & \beta_{1}=\gamma_{1} \gamma_{5}-\gamma_{2} \gamma_{4} \\
{ }_{227} & \beta_{2}=-q_{1} E \gamma_{5}+q_{2} E \gamma_{4} \\
{ }_{228} & \beta_{3}=q_{1} E \gamma_{2}-q_{2} E \gamma_{1} \\
{ }_{229} & \beta_{4}=-\alpha q_{1} E \gamma_{5}+\gamma_{2} \gamma_{3} \\
{ }_{230} & \beta_{5}=-q_{2} E \gamma_{3} \\
{ }_{231} & \beta_{6}=\alpha q_{1} q_{2} E^{2} \\
{ }_{232} & \beta_{7}=\alpha q_{1} E \gamma_{4}-\gamma_{1} \gamma_{3} \\
& \beta_{8}=q_{1} E \gamma_{3}
\end{array}
$$


${ }_{235}$ The determinant of $\frac{d \Phi}{d x}$ can be written

$$
\Gamma\left(x_{0}, x_{1}, x_{2}\right)=\operatorname{Det}\left(\frac{d \Phi}{d x}\right)=\left(c+a_{0} x_{0}+a_{1} x_{1}+a_{2} x_{2}\right) E^{3},
$$

where $c$ and $a_{i}$ are functions of the parameters. The map $\left(x_{0}, x_{1}, x_{2}\right) \mapsto \Gamma\left(x_{0}, x_{1}, x_{2}\right)$ is affine on the polyhedron $D$, hence it reaches its extrema on the vertexes of $D$. For a given set of parameters, it is then sufficient to compute the values of $\Gamma\left(x_{0}, x_{1}, x_{2}\right)$ on the vertexes of $D$ in order to see if $\Gamma\left(x_{0}, x_{1}, x_{2}\right)$ vanishes in $D$ or not.

We assume that the parameters are such that the map $\Phi$ is a diffeomorphism from $\stackrel{o}{D}$ to $\Phi(\stackrel{o}{D})$. This implies that system $(11)$ is observable.

In the new coordinates defined by $\left(z_{1}, z_{2}, z_{3}\right)^{T}=z=\Phi(x)=\left(h(x), L_{F} h(x), L_{F}^{2}(x)\right)^{T}$, our system can be written in the canonical form as follow:

$$
\left\{\begin{array}{l}
\dot{z}(t)=\underbrace{\left(\begin{array}{lll}
0 & 1 & 0 \\
0 & 0 & 1 \\
0 & 0 & 0
\end{array}\right)}_{A} z(t)+\left(\begin{array}{c}
0 \\
0 \\
\psi(z(t))
\end{array}\right) \\
y(t)=z_{1}(t)=\underbrace{(1,0, O)}_{C} z(t) .
\end{array}\right.
$$

where : $\psi(z)=L_{F}^{3} h\left(\Phi^{-1}(z)\right)=L_{F}^{3} h(x)=\varphi(x)$

The function $\varphi$ is smooth (it is a polynomial function of $x=\left(x_{0}, x_{1}, x_{2}\right)$ ) on the compact set $D$. Hence, it is globally Lipschitz on $D$. Therefore it can be extended by $\tilde{\varphi}$, a Lipschitz function on $\mathbb{R}^{3}$ which satisfies $\tilde{\varphi}(x)=\varphi(x)$, for all $x \in D$. In the same way we define $\tilde{\psi}$ the Lipschitz prolongation of the function $\psi$.

So we have the following system (13) defined on the whole space $\mathbb{R}^{3}$. The restriction of (13) to the domain D is the system (12):

$$
\left\{\begin{array}{l}
\dot{z}=A z+\left(\begin{array}{c}
0 \\
0 \\
\tilde{\psi}(z)
\end{array}\right) \\
y=C z
\end{array}\right.
$$

Hence, we have shown that system (11) satisfies the conditions of the following result which provides the observer construction.

Proposition 2.1 ([13]) Under the assumptions that

H1: $\Phi$ is a diffeomorphism from $\stackrel{o}{D}$ to $\Phi(\stackrel{o}{D})$. $(\stackrel{o}{D}$ is the interior of $D)$.

H2: $\varphi$ can be extended from $D$ to $\mathbb{R}^{3}$ by a $C^{\infty}$ function, globally Lipschitz on $\mathbb{R}^{3}$. 
Then an exponential observer for system (13) is given by the following system:

$$
\dot{\hat{z}}=A \hat{z}+\psi(\hat{z})+S^{-1}(\theta) C^{T}(y-C \hat{z}) .
$$

where $S(\theta)$ is the solution of

$$
0=-\theta S(\theta)-A^{T} S(\theta)-S(\theta) A^{T}+C^{T} C,
$$

and $\theta$ is large enough.

Here, $S(\theta)=\left(\begin{array}{ccc}\theta^{-1} & -\theta^{-2} & \theta^{-3} \\ -\theta^{-2} & 2 \theta^{-3} & -3 \theta^{-4} \\ \theta^{-3} & -3 \theta^{-4} & 6 \theta^{-5}\end{array}\right)$.

Precisely $\theta \geq 2 n c K \sqrt{S}$, where $K$ is the lipschitz coefficient of the function $\psi, n$ is the dimension of the space, and $S=\sup _{i, j}\left|S(1)_{i, j}\right|$.

For the proof one can see [13].

Going back to the our original system (9) via the transformation $\Phi^{-1}$, we have :

$$
\dot{\hat{x}}=\tilde{F}(\hat{x})+\left[\frac{d \Phi}{d x}\right]_{x=\hat{x}}^{-1} \times S(\theta)^{-1} C^{T}(y-h(\hat{x}))
$$

The restriction of this system to $D$ is the following system :

$$
\left\{\begin{aligned}
\dot{\hat{x}}_{0}= & -\alpha_{0} \hat{x}_{0}+\sum_{i=1}^{2} f_{i} l_{i} \hat{x}_{i}-\sum_{i=1}^{2} p_{i} \hat{x}_{i} \hat{x}_{0}-p_{0} \hat{x}_{0}^{2} \\
& +\left(3 \theta \beta_{1}+3 \theta^{2} \beta_{2}+\theta^{3} \beta_{3}\right)\left(y-q_{1} E \hat{x}_{1}-q_{2} E \hat{x}_{2}\right) \\
\dot{\hat{x}}_{1}= & \alpha \hat{x}_{0}-\left(\alpha_{1}+q_{1} E\right) \hat{x}_{1} \\
& +\left(3 \theta \beta_{4}+3 \theta^{2} \beta_{5}+\theta^{3} \beta_{6}\right)\left(y-q_{1} E \hat{x}_{1}-q_{2} E \hat{x}_{2}\right) \\
\dot{\hat{x}}_{2}= & \alpha \hat{x}_{1}-\left(\alpha_{2}+q_{2} E\right) \hat{x}_{2} \\
& +\left(3 \theta \beta_{7}+3 \theta^{2} \beta_{8}+\theta^{3} \beta_{9}\right)\left(y-q_{1} E \hat{x}_{1}-q_{2} E \hat{x}_{2}\right)
\end{aligned}\right.
$$

which is the observer for the fishery model (9). This observer is particularly simple since it is only a copy of (9), together with a corrective term depending on $\theta$.

\subsection{Simulations and comments}

We present here some simulation results that show the efficiency of the observer of system (9). The simulations have been done with the free software SCILAB.

Remarque 2.1 For the simulations we extend the function $\varphi$ by continuity in order to make it globally lipschitz on $\mathbb{R}^{3}$ in the following way: We denote $\tilde{\varphi}$ the prolongation of $\varphi$ to $\mathbb{R}^{3}$ and the function $\pi$ the projection on the domain $D$ and we construct $\tilde{\varphi}=\varphi \circ \pi$. The extended function $\tilde{\varphi}$ has the same Lipschitz coefficient as $\varphi$. The projection $\pi$ is defined as follows: for $x \in \mathbb{R}^{3}, \pi(x)=\bar{x}$, where $\bar{x} \in D$ is such that $\operatorname{dist}(x, D)=\|x-\bar{x}\|$, i.e., $\bar{x}$ satisfies $\|x-\bar{x}\|=\min _{u \in D}\|u-x\|$. The extension algorithm is described in Appendix B.. 
We use the following fishery parameters [36], [43].

$$
\begin{aligned}
& \alpha_{0}=1.3 ; \alpha_{1}=0.9 \\
& \alpha_{2}=0.85 ; p_{0}=0.2 \\
& p_{1}=0.1 ; p_{2}=0.1 ; \\
& q_{1}=0.07 ; q_{2}=0.15 \\
& f_{1}=0.5 ; f_{2}=0.5 \\
& l_{1}=10 ; l_{2}=10 \\
& E=0.5 ; \alpha=0.8
\end{aligned}
$$

For these parameter the Jacobian of the function $\Phi$ is expressed as:

$$
\frac{d \Phi}{d x}=\left(\begin{array}{ccc}
0 & 0.035 & 0.075 \\
0.028 & 0.027275 & -0.069375 \\
-0.01458-0.0112 x_{0} & 0.0589979-0.0028 x_{0} & 0.191338-0.0028 x_{0} \\
-0.0028 x_{1}-0.0028 x_{2} & &
\end{array}\right)
$$

The determinant of this matrix is:

$$
\operatorname{Det}\left(\frac{d \Phi}{d x}\right)=1.612 \times 10^{-6}+0.00004697 x_{0}+0.0000125265 x_{1}+0.0000125265 x_{2} .
$$

The states $x_{0}, x_{1}$ and $x_{2}$ are time varying but remain in the positive orthant; so the $\operatorname{Det}\left(\frac{d \Phi}{d x}\right)$ does not vanish. Therefore $\frac{d \Phi}{d x}$ is invertible and then $\Phi(x)$ is a diffeomorphism.

With the parameters defined in the top of this section, we compute the coordinates of the higher corner $B$ of the parallelepiped $D([43])$ and we get $B=$ $(25 ; 20.639 ; 17.868)$.

The nontrivial equilibrium point is $x^{*}=(18.572 ; 15.89 ; 13.743)$.

The construction of the high gain observer (15) is done with $\theta=17$. For the simulations we have taken $x(0)=[21 ; 20 ; 15]$ and $\hat{x}(0)=[35 ; 40 ; 10]$.

Comments: Using the same parameters values, when we do not use the Lipschitz prolongation of the function $\varphi$ to the whole $\mathbb{R}^{3}$, the state estimation $\hat{x}(t)$ computed by the observer tends to infinity in finite time. This actually happens in the beginning of the integration process as it can be seen in Figures 2, 4 and 6 . When the Lipschitz prolongation of the function $\varphi$ to the whole $\mathbb{R}^{3}$ is done, the convergence of the estimates delivered by the observer is quite fast (Figures 3, 5 and 7 ).

\section{A global model}

\subsection{The model and the observer}

Here we consider the dynamics of a fish population moving between two zones (see [9]). The first zone is a free fishing area, and the second zone is a reserve area 
330 Therefore $\frac{d \Phi}{d x}=\left(\begin{array}{cc}q E & 0 \\ r_{1} q E-\frac{2 r_{1} q E x_{1}}{K_{1}}-\left(m_{12}+q E\right) q E & m_{21} q E\end{array}\right)$

$r_{1}$ and $r_{2}$ represent the intrinsic growth of each fish sub-population, respectively, $K_{1}$ and $K_{2}$ are the carrying capacities of fish species in the unreserved and reserved areas, respectively; $q$ is the catchability coefficient of fish species in the unreserved area. The parameters $r_{1}, r_{2}, q, m_{12}, m_{21}, K_{1}$ and $K_{2}$ are positives constants.

To the system (17) we associate the capture (i.e. the output) $y=q E x_{1}$ (the total of caught fish in the unreserved area), with this output, we show the observability condition of system (17) and construct an auxiliary system that will give a dynamical estimation of the state of system (17).

It is possible to find a positive real number $w_{0}$ in such a way that for any $w \geq w_{0}$ the following compact set $D_{w}$ is positively invariant for system (17). This compact set is given

$$
D_{w}=\left\{\left(x_{1}, x_{2}\right) \in \mathbb{R}_{+}^{2}: x_{1}+x_{2} \leq w\right\},
$$

The proof of this fact as well as the computation of $w_{0}$ as a function of the parameters are given in Appendix A..

Let us denote by $f$ the vector field that defines the system (17):

$$
f(x)=\left(\begin{array}{c}
r_{1} x_{1}\left(1-\frac{x_{1}}{K_{1}}\right)-\left(m_{12}+q E\right) x_{1}+m_{21} x_{2} \\
r_{2} x_{2}\left(1-\frac{x_{2}}{K_{2}}\right)+m_{12} x_{1}-m_{21} x_{2}
\end{array}\right) .
$$

Let $z=\left(\begin{array}{c}z_{1} \\ z_{2}\end{array}\right), y(t)=h(x)=q E x_{1}(t)$ and

${ }_{329} \Phi(x)=\left(\begin{array}{c}y \\ \dot{y}\end{array}\right)=\left(\begin{array}{c}q E x_{1} \\ r_{1} q E x_{1}\left(1-\frac{x_{1}}{K_{1}}\right)-\left(m_{12}+q E\right) q E x_{1}+m_{21} q E x_{2}\end{array}\right)$. 
331 and $\operatorname{Det}\left(\frac{d \Phi}{d x}\right)=q^{2} E^{2} m_{21}$.

332 As the parameters $q, E$ and $m_{21}$ are positive $(\neq 0)$, we can conclude that $\operatorname{Det}\left(\frac{d \Phi}{d x}\right) \neq$ з3з 0 , and then, $\Phi$ is a diffeomorphism from $\mathbb{R}^{2}$ to $\Phi\left(\mathbb{R}^{2}\right)$, thus system (17) is observable. 334 Thanks to ([13]) the observer can be expressed as follows:

$$
\dot{\hat{x}}=\tilde{f}(\hat{x})+\left(\frac{d \Phi}{d x}\right)^{-1} \times S(\theta)^{-1} C^{T}(y-h(\hat{x})),
$$

$337 \quad S(\theta)^{-1}=\left(\begin{array}{cc}2 \theta & \theta^{2} \\ \theta^{2} & \theta^{3}\end{array}\right)$, with $\theta \geq 1$.

338

339 the whole $\mathbb{R}^{2}$ space, $C=(1,0)$ and equations:

\subsection{Simulation} the following parameters :

$r_{1}=\frac{7}{10} ; r_{2}=\frac{5}{10}$,

$q=\frac{25}{100}, E=\frac{9}{10}$,

$K_{1}=10, K_{2}=\frac{22}{10}$,

$m_{12}=\frac{2}{10}, m_{21}=\frac{1}{10}$, in Figures 9 and 11.

where $\tilde{f}$ is a Lipschitz extension of the function $f$ from the invariant domain $D_{w}$ to

The restriction of the estimator (18) to the invariant domain $D_{w}$ is given by the

$$
\left\{\begin{array}{l}
\dot{\hat{x}}_{1}=r_{1} \hat{x}_{1}\left(1-\frac{\hat{x}_{1}}{K_{1}}\right)-m_{12} \hat{x}_{1}+m_{21} \hat{x}_{2}-q E \hat{x}_{1}+2 \theta\left(x_{1}-\hat{x}_{1}\right) \\
\dot{\hat{x}}_{2}=r_{2} \hat{x}_{2}\left(1-\frac{\hat{x}_{2}}{K_{2}}\right)+m_{12} \hat{x}_{1}-m_{21} \hat{x}_{2} \\
\quad+2 \theta\left(\frac{q E}{m_{21}}-\frac{1}{m_{21}}+\frac{m_{12}}{q E m_{21}}+\frac{2 r_{1} x_{1}}{m_{21}}+\frac{\theta}{m_{21}}\right)\left(x_{1}-\hat{x}_{1}\right),
\end{array}\right.
$$

Simulations for the model (17) together with its observer (18) have been done with

Thanks to formula (20) we compute $w_{0}=8.987$ and we take $w=20$.

With these parameters, the invariant domain is the triangle defined by $O(0,0)$, $A(w, 0)=A(20,0)$ and $B(0, w)=B(0,20)$, and we take $\theta=4$.

Using the SCILAB free software, the time evolution of the states as well as the respective estimates when the Lipschitz extension is done are drawn in Figures 8 and 10. When the Lipschitz extension has not been done, the simulations are given 


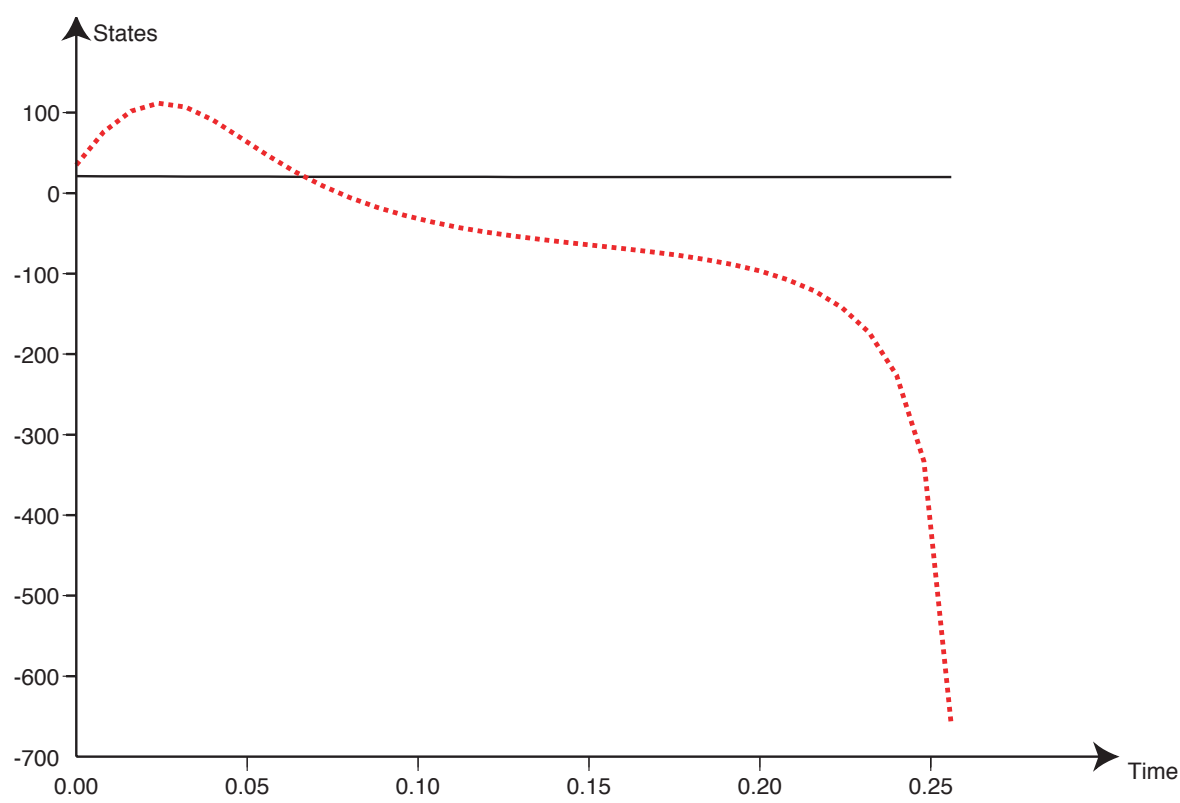

Figure 2: Simulation of system (9) with its observer (15): $x_{0}$ (solid line) and its estimate $\hat{x}_{0}$ (dashed line) when $\varphi$ is not extended

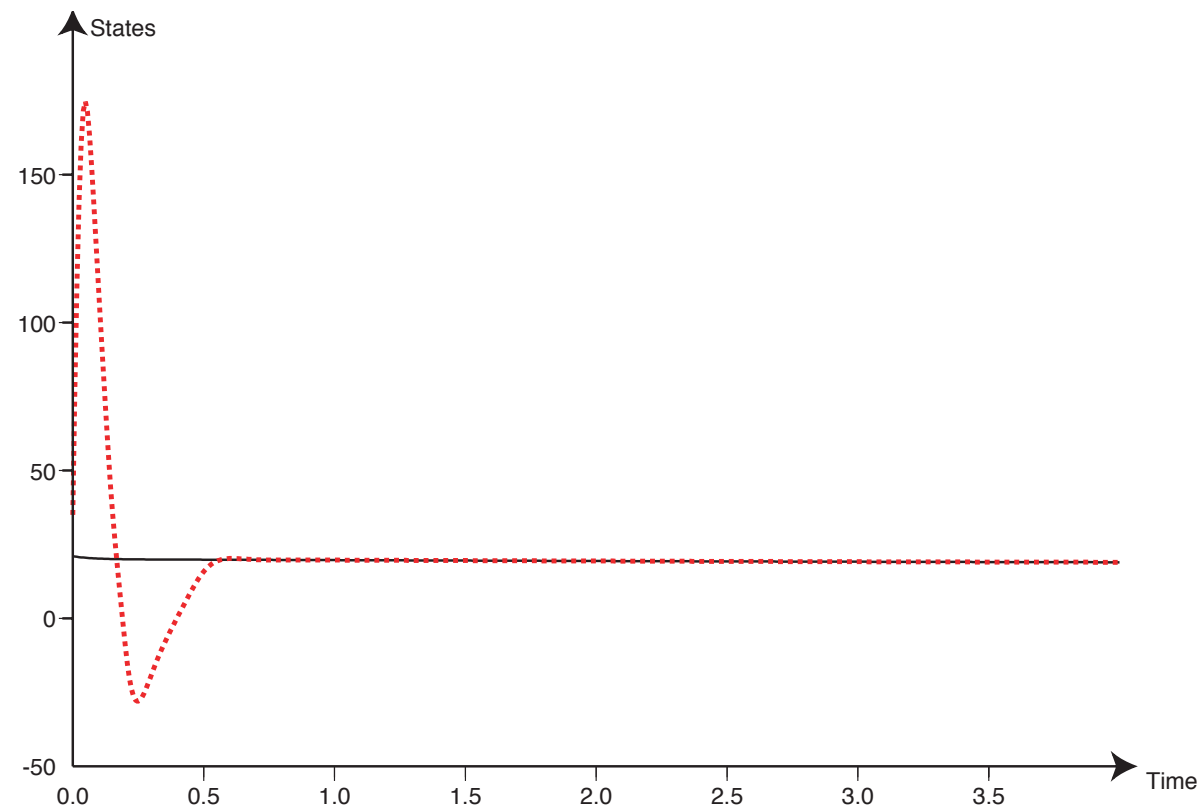

Figure 3: Simulation of system (9) with its observer (15): $x_{0}$ (solid line) and its estimate $\hat{x}_{0}$ (dashed line) when $\varphi$ is extended 


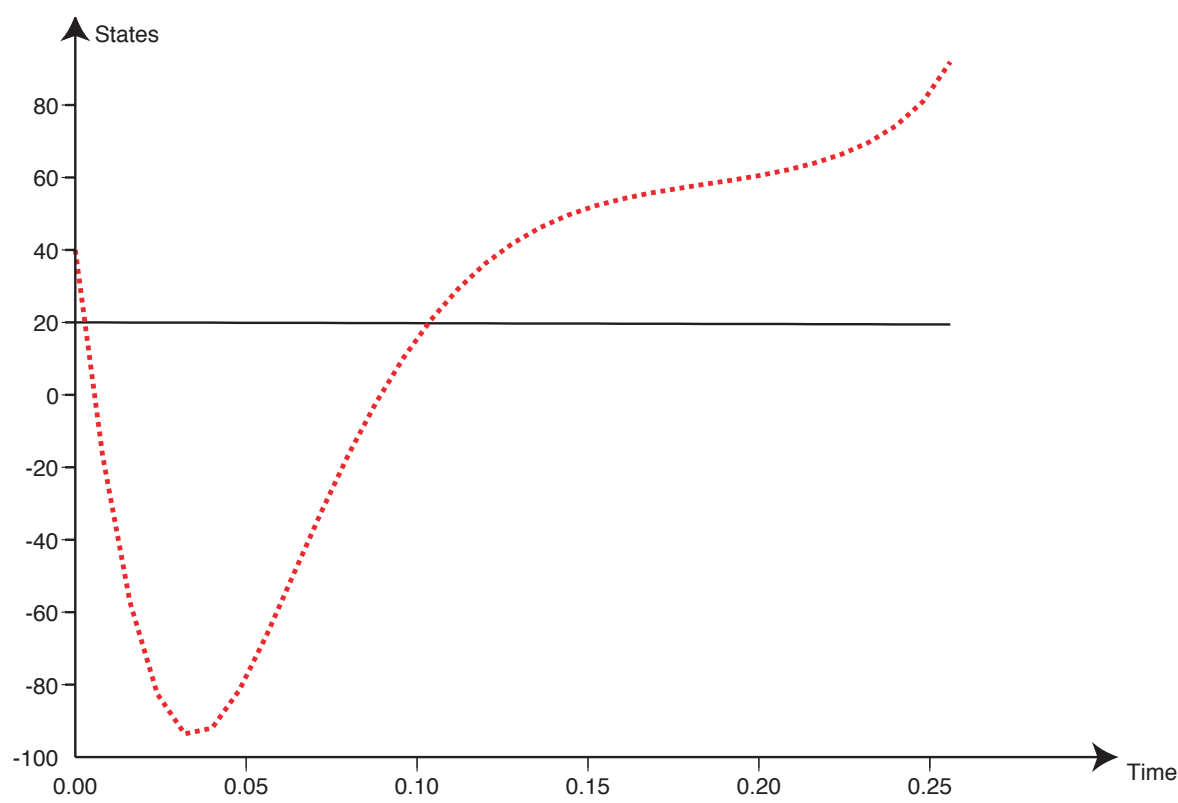

Figure 4: Simulation of system (9) with its observer (15): $x_{1}$ (solid line) and its estimate $\hat{x}_{1}$ (dashed line) when $\varphi$ is not extended

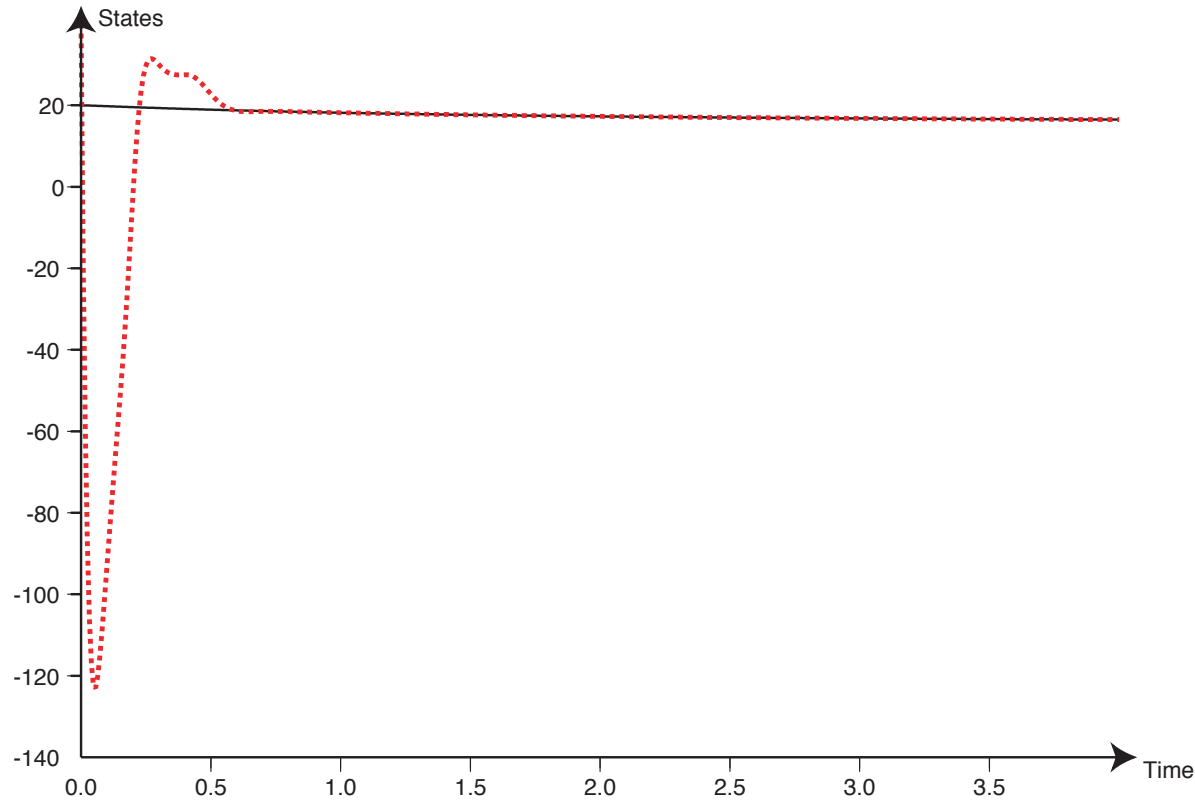

Figure 5: Simulation of system (9) with its observer (15): $x_{1}$ (solid line) and its estimate $\hat{x}_{1}$ (dashed line) when $\varphi$ is extended 


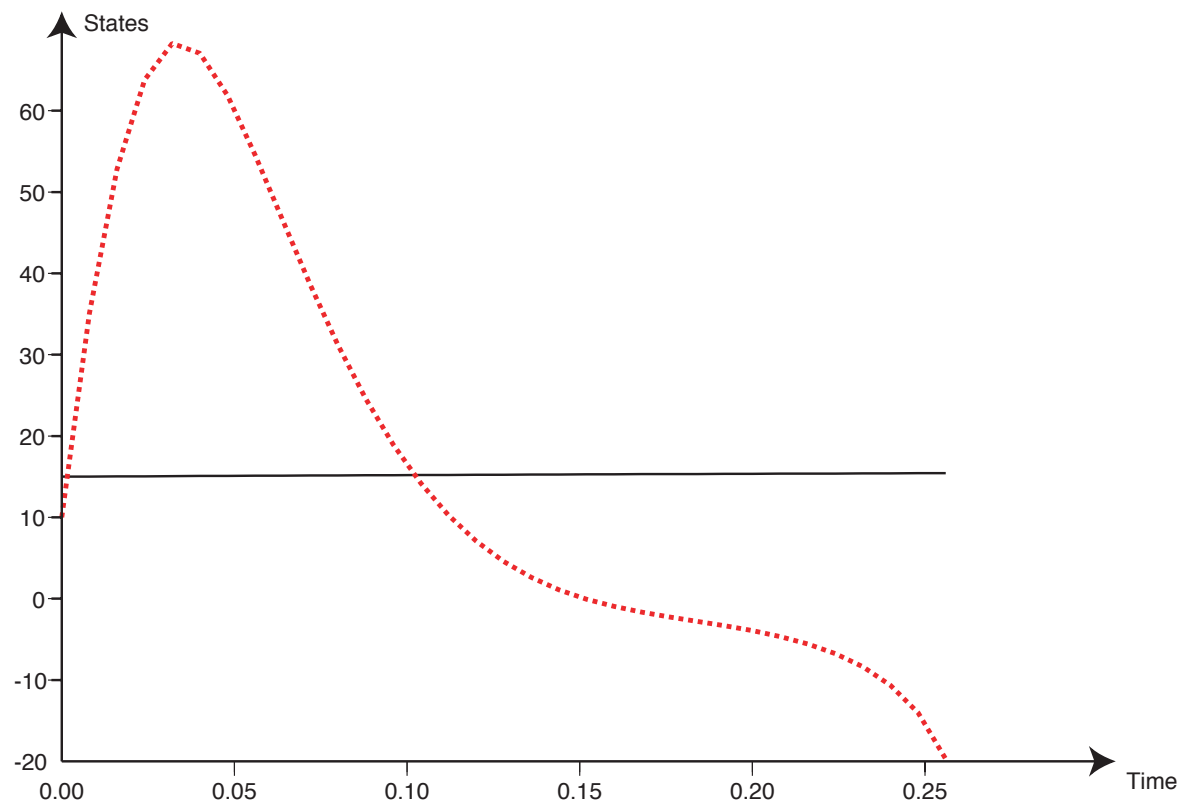

Figure 6: Simulation of system (9) with its observer (15): $x_{2}$ (solid line) and its estimate $\hat{x}_{2}$ (dashed line) when $\varphi$ is not extended

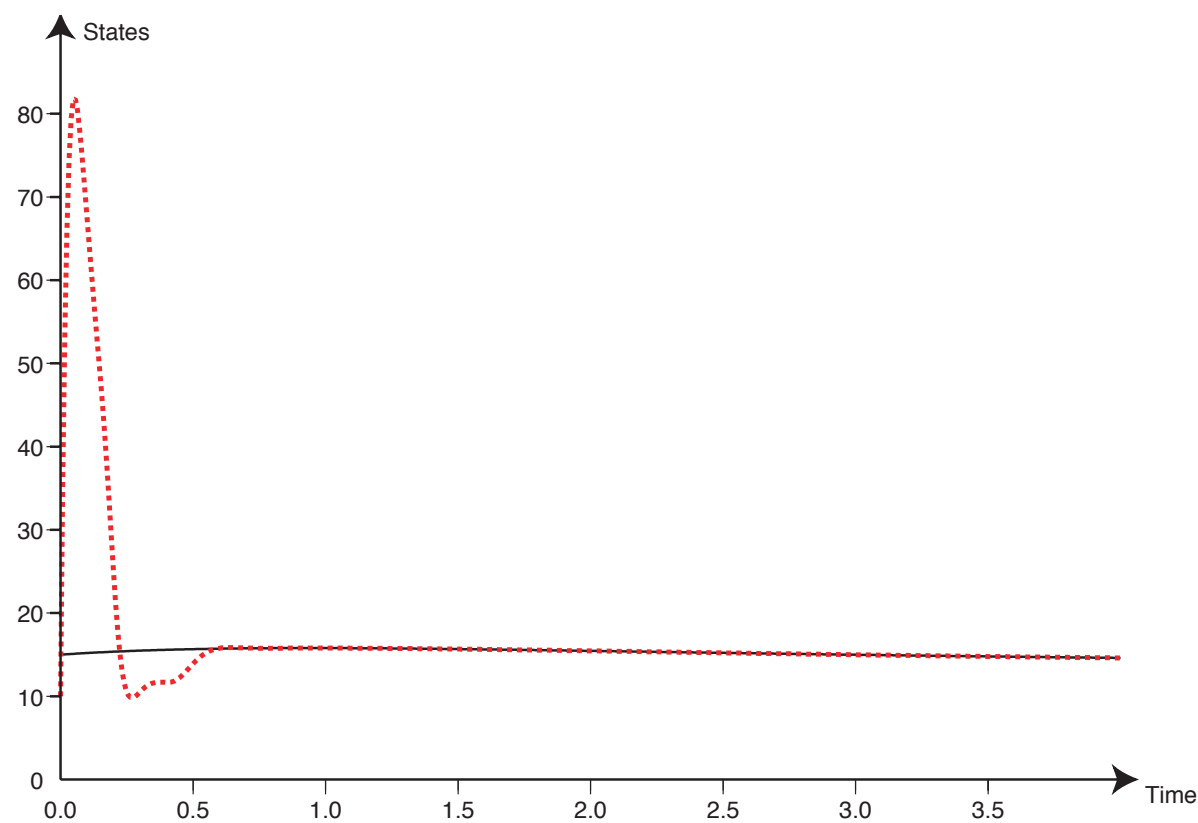

Figure 7: Simulation of system (9) with its observer (15): $x_{2}$ (solid line) and its estimate $\hat{x}_{2}$ (dashed line) when $\varphi$ is extended 


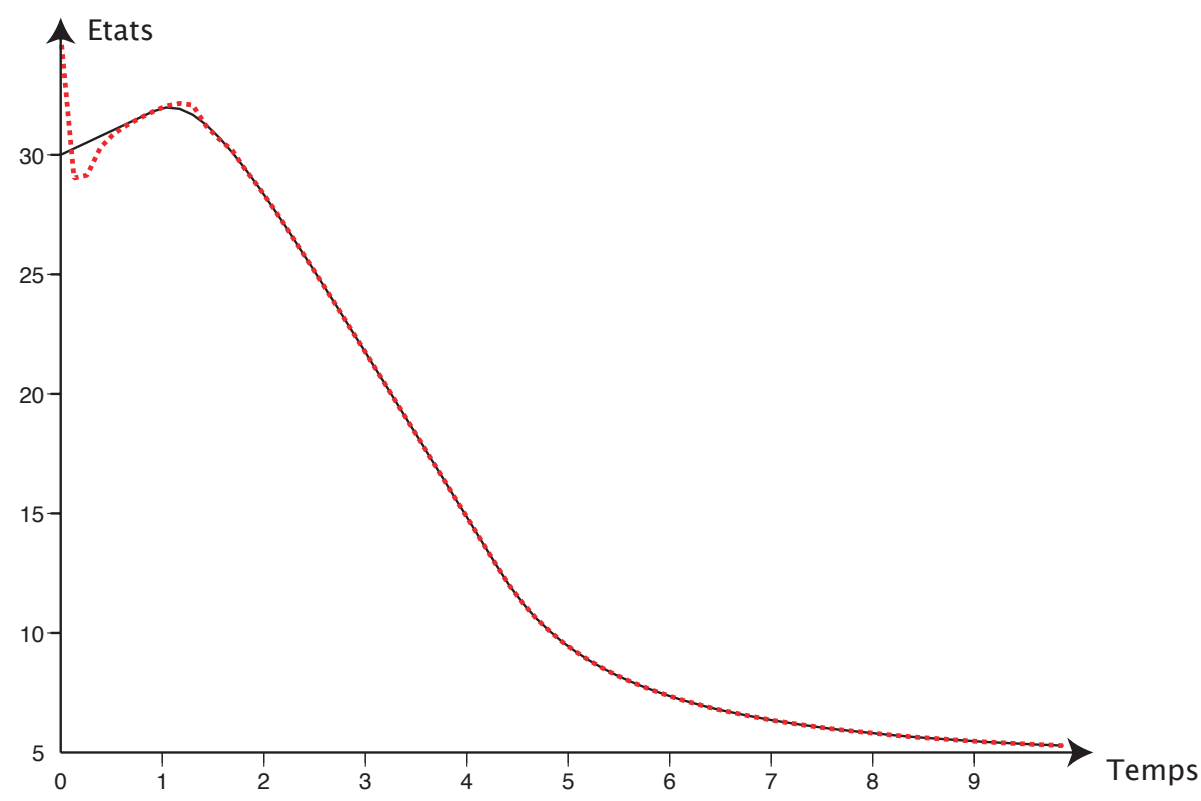

Figure 8: Simulation of system (17) with its observer (18): $x_{1}$ (solid line) and its estimate $\hat{x}_{1}$ (dashed line) when $f$ is extended

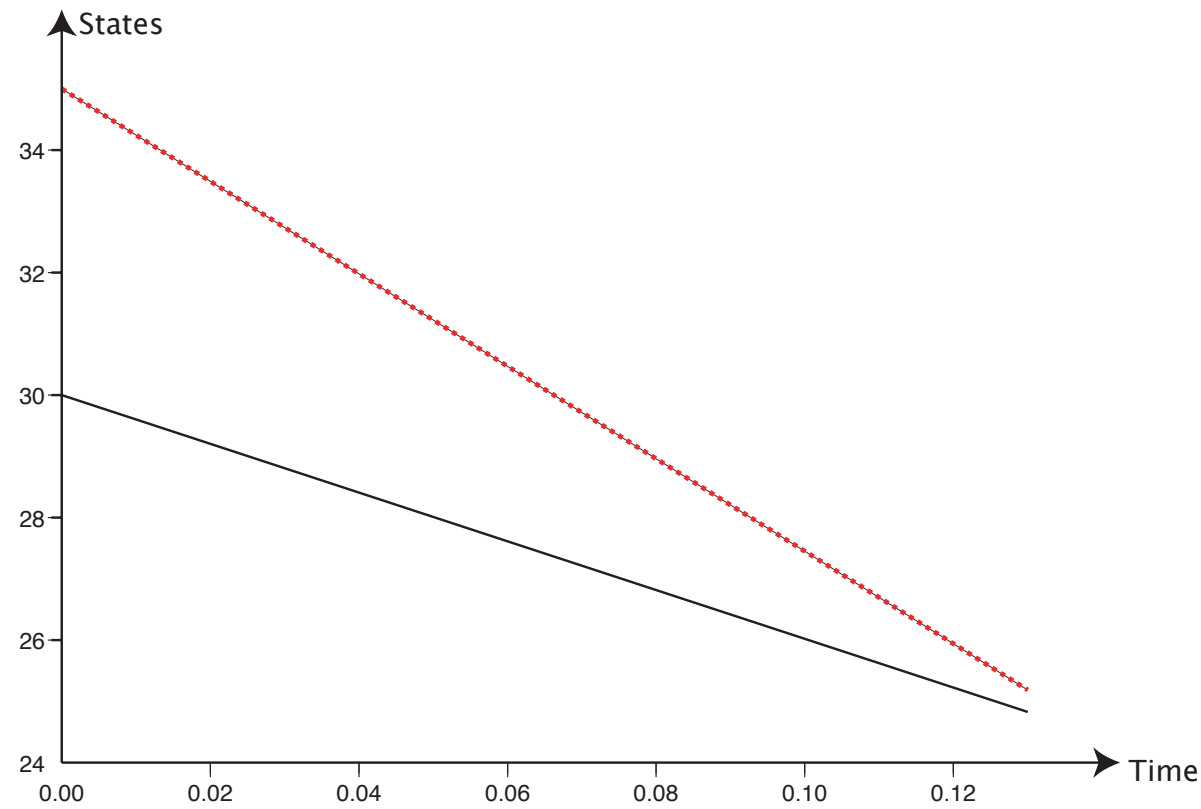

Figure 9: Simulation of system (17) with its observer (18): $x_{1}$ (solid line) and its estimate $\hat{x}_{1}$ (dashed line) when $f$ is not extended 


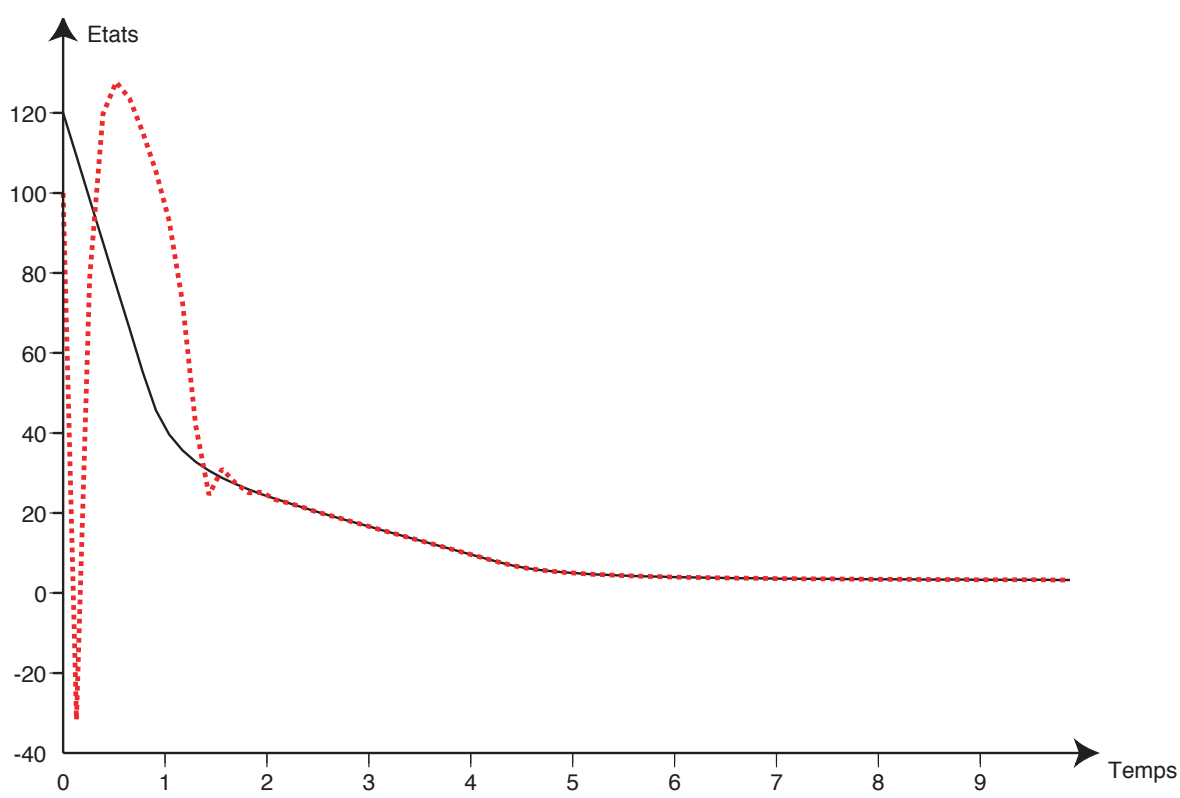

Figure 10: Simulation of system (17) with its observer (18): $x_{2}$ (solid line) and its estimate $\hat{x}_{2}$ (dashed line) when $f$ is extended

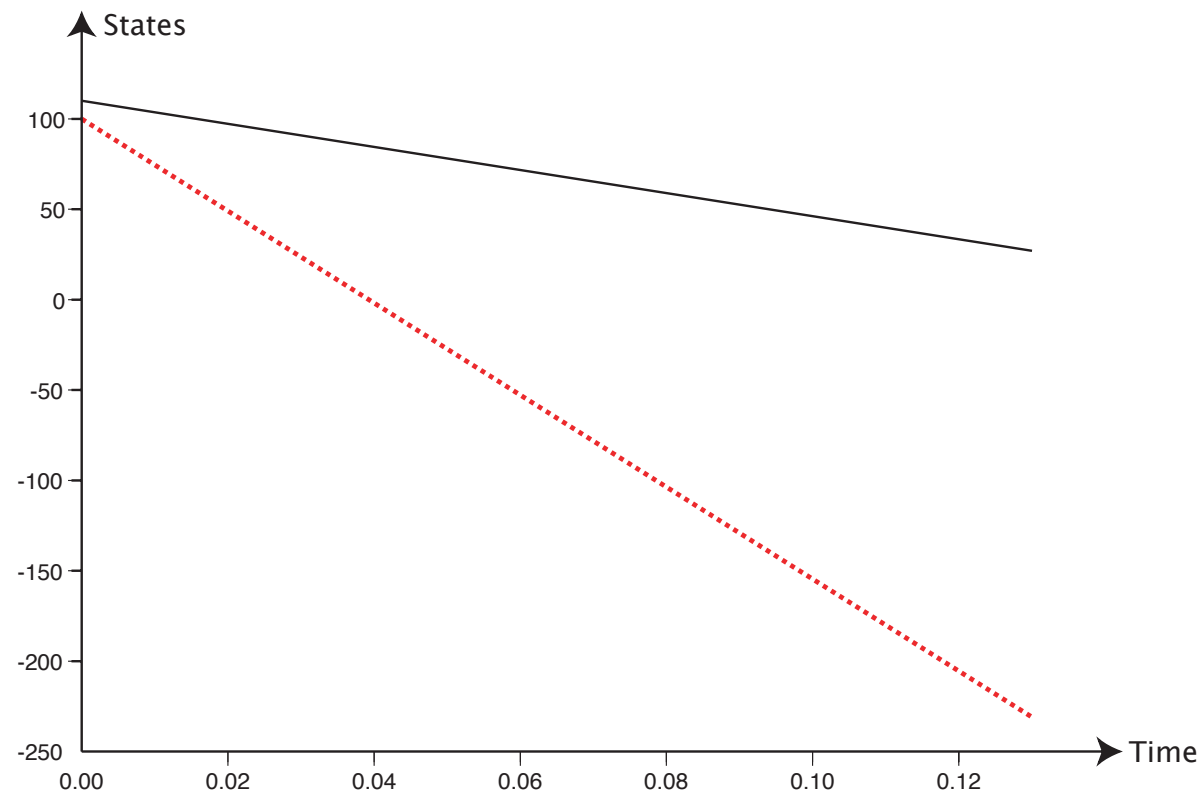

Figure 11: Simulation of system (17) with its observer (18): $x_{2}$ (solid line) and its estimate $\hat{x}_{2}$ (dashed line) when $f$ is not extended 


\section{Conclusion}

We have tried to combine modern Control Theory, Computer Science and Mathematics to address the state estimation problem for systems that model the dynamics of fish populations submitted to a fishing action. Indeed one of the important problems in fishery sciences is to estimate the state of the resource using the available data, in order to produce scientific opinions that can be helpful for developing management policies that need to have a good estimate of the available resource.

In this work, we have constructed High Gain observers for some fishery models. With the use of judicious value of the gain parameter $\theta$ we obtain satisfactory estimation of the real state. The observer's convergence is quite fast and does not depend on the initial conditions choice. Therefore one can get a "good" estimate of the unmeasurable real state very quickly. It is interesting to notice that the state estimator built in this paper for the stage-structured model use only the total catch to give not only an estimate of the total stock but also an estimate of the number of individuals in each stage class. The classical techniques like the Cohort Analysis (CA) or the Virtual Population Analysis (VPA) use the total catch for each stageclass in order to give estimates of the number of individuals in each stage class. In practice it is easier to measure the total catch 9without doing any distinction between individuals) then to measure the catch for each stage class. However the observers given in this paper assume that the model is good enough and that the parameters values are available.

Nonlinear control techniques are useful for studying and controlling complex systems. Although they have been initially developed for mechanical and electrical systems their applications to biological and environmental problems are growing. Tools of optimal control theory have been extensively used in renewable resource management ([8], [3] [23] [18] [25],[31],[45],[12],[32]). The present paper shows that the estimation problem in fisheries management can also be investigated from the point of view of control engineering.

\section{Acknowledgments}

We thank the anonymous referees for their valuable comments and suggestions that have helped us to improve the presentation of this article.

\section{References}

[1] V. Alcaraz-Gonzalez, R. Salazar-Pena, V. Gonzalez-Alvarez, J.-L. Gouze, and J.-P. Steyer. A tunable multivariable nonlinear robust observer for biological systems. Comptes Rendus Biologies, 328(4):317-325, 2005.

[2] M. Alamir and L. Calvillo-Corona. Further results on nonlinear recedinghorizon observers. IEEE Transactions on Automatic Control, 47(7):1184-1188, Jul 2002. 
[3] S. Aniţa. Optimal harvesting for a nonlinear age-dependent population dynamics. J. Math. Anal. Appl., 226(1):6-22, 1998.

[4] A. Atassi and H. Khalil. Separation results for the stabilization of nonlinear systems using different high-gain observer designs. Syst. Control Lett., 39(3):183-191, 2000.

[5] O. Bernard, G. Sallet, and A. Sciandra, Nonlinear observers for a class of biological systems: application to validation of a phytoplanktonic growth model, IEEE Trans. Autom. Control, 43 (1998), 1056-1065.

[6] D. Bestle and M. Zeitz. Canonical form observer design for non-linear timevariable systems. International Journal of Control, 38(2):419 - 431, 1983.

[7] G. Bornard and H. Hammouri. A high gain observer for a class of uniformly observable systems. Proceedings of the 30th IEEE Conference on Decision and Control, 1991., pages 1494-1496 vol.2, Dec 1991.

[8] C. W. Clark. Mathematical bioeconomics. The optimal management of renewable resources. 2nd ed. Wiley-Interscience Publication. New York, 1990.

[9] B. Dubey, P. Chandra, and P. Sinha. A model for fishery resource with reserve area. Nonlinear Anal., Real World Appl., 4(4):625-637, 2003.

[10] M. Farza, K. Busawon, and H. Hammouri, Simple nonlinear observers for online estimation of kinetic rates in bioreactors, Automatica, 34 (1998), 301-318.

[11] M. Gámez, I. López, and S. Molnár. Monitoring environmental change in an ecosystem. Biosystems, 93(3):211-217, 2008.

[12] S. Gao, L. Chen, and L. Sun. Optimal pulse fishing policy in stage-structured models with birth pulses. Chaos, Solitons \& Fractals, 25(5):1209-1219, 2005.

[13] J. P. Gauthier, H. Hammouri, and S. Othman, A simple observer for nonlinear systems applications to bioreactors, IEEE Trans. Autom. Control, 37 (1992), 875-880.

[14] J. P. Gauthier and I. Kupka. Observability and observers for nonlinear systems. SIAM J. Control Optimization, 32(4):975-994, 1994.

[15] J. P. Gauthier, I. Kupka. Deterministic observation Theory and Applications. Cambridge University Press, 2001.

[16] A. Guiro, A. Iggidr, D. Ngom, and H. Touré. A Non Linear Observer for a Fishery Model. In Proc. 17th Triennial IFAC World Congress, Seoul, Korea, July 6-11, 2008.

[17] J.A. Gulland, Fish Stock Assessment, a manual of basic methods, Wiley, Chichester (UK), 1983. 
[18] J. W. Horwood and P. Whittle. The optimal harvest from a multicohort stock. IMA J. Math. Appl. Med. Biol., 3(2):143-155, 1986.

[19] X. Hulhoven, A. V. Wouwer, and P. Bogaerts. Hybrid extended luenbergerasymptotic observer for bioprocess state estimation. Chemical Engineering Science, 61(21):7151-7160, 2006.

[20] A. Iggidr, Controllability, observability and stability of mathematical models, in Mathematical Models. In Encyclopedia of Life Support Systems (EOLSS). Ed. Jerzy A. Filar. Developed under the auspices of the UNESCO, Eolss Publishers, Oxford,UK, [http://www.eolss.net].

[21] A. Iggidr and G. Sallet. Exponential stabilization of nonlinear systems by an estimated state feedback. In Proc. of the 2nd European Control Conference ECC'93, Groningen, Pays-Bas., 1993.

[22] A. Isidori. Nonlinear control systems. 3rd ed. Communications and Control Engineering Series. Berlin: Springer., 1995.

[23] O. L. R. Jacobs, D. J. Ballance, and J. W. Horwood. Fishery management as a problem in feedback-control. AUTOMATICA, 27(4):627-639, Jul 1991.

[24] W. Kang. Moving horizon numerical observers of nonlinear control systems. IEEE Trans. Automat. Control, 51(2):344-350, 2006.

[25] T. K. Kar. Management of a fishery based on continuous fishing effort. Nonlinear Analysis: Real World Applications, 5(4):629-644, 2004.

[26] G. Kreisselmeier and R. Engel, Nonlinear observers for autonomous Lipschitz continuous systems, IEEE Trans. Automat. Control, 48 (2003), 451-464.

[27] A. J. Krener and W. Respondek, Nonlinear observers with linearizable error dynamics, SIAM Journal on Control and Optimization, 23 (1985),197-216.

[28] A. J. Krener and M. Xiao, Nonlinear observer design in the Siegel domain, SIAM J. Control Optimization, 41 (2002), 932-953.

[29] I. López, M. Gámez, J. Garay, and Z. Varga. Monitoring in a lotka-volterra model. Biosystems, 87(1):68-74, 2007.

[30] D. G. Luenberger, An introduction to observers, IEEE Trans. Automat. Control, 16 (1971), 596-602.

[31] T. Marutani. On the optimal path in the dynamic pool model for a fishery. Reviews in Fish Biology and Fisheries, 18(2):133-141, 2008.

[32] R. Mchich, N. Charouki, P. Auger, N. Raissi, and O. Ettahiri. Optimal spatial distribution of the fishing effort in a multi fishing zone model. Ecological Modelling, 197(3-4):274-280, 2006. 
[33] H. Michalska and D. Mayne. Moving horizon observers and observer-based control. Automatic Control, IEEE Transactions on, 40(6):995-1006, Jun 1995.

[34] P. Moraal and J. Grizzle. Observer design for nonlinear systems with discretetime measurements. IEEE Trans. Autom. Control, 40(3):395-404, 1995.

[35] D. Ngom, A. Iggidr, A. Guiro, A. Ouahbi An observer for a nonlinear agestructured model of a harvested fish population Mathematical Biosciences and Engineering 5(2):337-354, april 2008.

[36] A. Ouahbi, A. Iggidr, M. El Bagdouri. Stabilization of an exploited fish population. Systems Analysis Modelling simulation, 43:513-524, 2003.

[37] M. Xiao, N. Kazantzis, C. Kravaris, and A. J. Krener, Nonlinear discrete-time observer design with linearizable error dynamics, IEEE Trans. Autom. Control, 48 (2003), 622-626.

[38] A. Rapaport and A. Maloum. Design of exponential observers for nonlinear systems by embedding. Int. J. Robust Nonlinear Control, 14(3):273-288, 2004.

[39] E. D. Sontag, Mathematical control theory. Deterministic finite-dimensional systems, volume 6 of Texts in Applied Mathematics. Springer-Verlag, New York, 1998.

[40] F. E. Thau. Observing the state of non-linear dynamic systems. International Journal of Control, 17(3):471 - 479, 1973.

[41] A. Tornambe. Use of asymptotic observers having-high-gains in the state and parameter estimation. Proceedings of the 28th IEEE Conference on Decision and Control, 1989., pages 1791-1794 vol.2, Dec 1989.

[42] L. A. Torres, V. Ibarra-Junquera, P. Escalante-Minakata, and H. C. Rosu. High-gain nonlinear observer for simple genetic regulation process. Physica AStatistical Mechanics and its Applications, 380:235-240, 2007.

[43] S. Touzeau. Modèles de contrôle en gestion des pêches. Thesis, University of Nice-Sophia Antipolis, France, 1997.

[44] S. Touzeau and J.-L. Gouzé. On the stock-recruitment relationships in fish population models. Environmental Modeling and Assessment, 3:87-93, 1998.

[45] C. White and B. E. Kendall. A reassessment of equivalence in yield from marine reserves and traditional fisheries managament. Oikos, 116(12):2039-2043, 2007.

[46] M. Zeitz. The extended Luenberger observer for nonlinear systems. Syst. Control Lett., 9:149-156, 1987.

[47] G. Zimmer, State observation by on-line minimization, Int. J. Control, 60 (1994), 595-606. 


\section{Appendix A. Positive invariance of $D_{w}$}

Let $N=x_{1}+x_{2}$.

$\dot{N}=-q E x_{1}+r_{1}\left(1-\frac{x_{1}}{K_{1}}\right) x_{1}+\left(N-x_{1}\right)\left(1-\frac{N-x_{1}}{K_{2}}\right) r_{2}$

Let $w$ be a positive real number, for $N=w$, we have

$\dot{N}=-q E x_{1}+r_{1}\left(1-\frac{x_{1}}{K_{1}}\right) x_{1}+\left(w-x_{1}\right)\left(1-\frac{w-x_{1}}{K_{2}}\right) r_{2}=g\left(x_{1}\right)$

The function $g$ is defined for $0 \leq x_{1} \leq w$.

$g(0)=w\left(1-\frac{w}{K_{2}}\right) r_{2}$

$g(w)=-q u w+w\left(1-\frac{w}{K_{1}}\right) r_{1}$

$g^{\prime}\left(x_{1}\right)=r_{1}-r_{2}-q u+\frac{2 w r_{2}}{K_{2}}-2\left(\frac{r_{1}}{K_{1}}+\frac{r_{2}}{K_{2}}\right) x_{1}$

$g^{\prime}\left(x_{1}\right)=0 \Leftrightarrow x_{1}=\bar{x}_{1}=\frac{K_{1}\left(K_{2} r_{1}-K_{2} r_{2}-q u K_{2}+2 w r_{2}\right)}{2\left(K_{2} r_{1}+K_{1} r_{2}\right)}$

The maximum value of the function $g$ is then given by the expression

$\frac{K_{1} K_{2}\left(q u-r_{1}+r_{2}\right)^{2}+\left(4 K_{2} r_{1} r_{2}+K_{1}\left(-4 q u r_{2}+4 r_{1} r_{2}\right)\right) w-4\left(r_{1} r_{2}\right) w^{2}}{4\left(K_{2} r_{1}+K_{1} r_{2}\right)}$

It is therefore clear that this maximum is non positive if $w \geq w_{0}$ with

$$
w_{0}=\frac{r_{1} r_{2}\left(K_{1}+K_{2}\right)-q u K_{1} r_{2}+\sqrt{r_{2}\left(K_{2} r_{1}+K_{1} r_{2}\right)\left(K_{1}\left(-q u+r_{1}\right)^{2}+K_{2} r_{1} r_{2}\right)}}{2 r_{1} r_{2}}
$$

This shows that for any real number $w \geq w_{0}$, the compact set

$$
D_{w}=\left\{\left(x_{1}, x_{2}\right) \in \mathbb{R}_{+}^{2}: x_{1}+x_{2} \leq w\right\}
$$

is positively invariant for system (17).

\section{Appendix B. Construction of the Lipschitz exten- sion of $\varphi$}

The function $\varphi$ is Lipschitz on the compact set $\mathrm{D}=\left[a_{0}, b_{0}\right] \times\left[a_{1}, b_{1}\right] \times\left[a_{2}, b_{2}\right]$. Our aim is to extend it to a function $\tilde{\varphi}$ which is Lipschitz with the same Lipschitz coefficient in the whole $\mathbb{R}^{3}$.

Let $a\left(a_{0}, a_{1}, a_{2}\right)$, (respectively $b\left(b_{0}, b_{1}, b_{2}\right)$ ), the lower corner, (respectively the upper corner) of the domain $\mathrm{D}$ and $x\left(x_{0}, x_{1}, x_{2}\right)$ an unspecified point of $\mathbb{R}^{3}$.

The problem of the extension is set for point $x \notin \mathrm{D}$; in this situation we have 26 possibilities according to the situation of $x$. The different situations correspond to $x_{i} \leq a_{i}, a_{i} \leq x_{i} \leq b_{i}$, or $x_{i} \geq b_{i}$.

The principle of this prolongation is to compose the function $\varphi$ with the function $\pi$ (the projection function of the point $x$ on the domain $\mathrm{D}$ ).

The extension of function $\varphi$ is described by the following algorithm:

if $x_{0} \leq a_{0}$ then

if $x_{1} \leq a_{1}$ then 


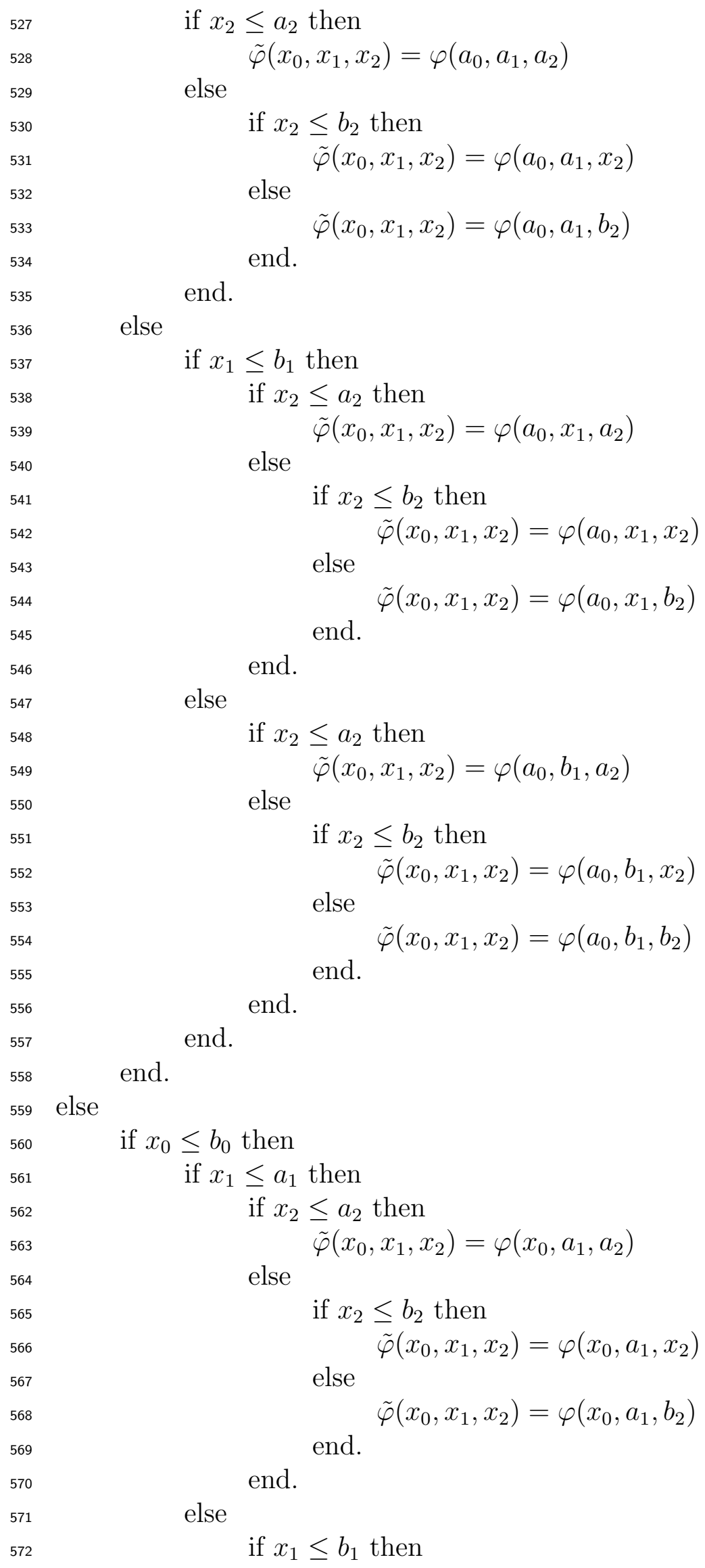




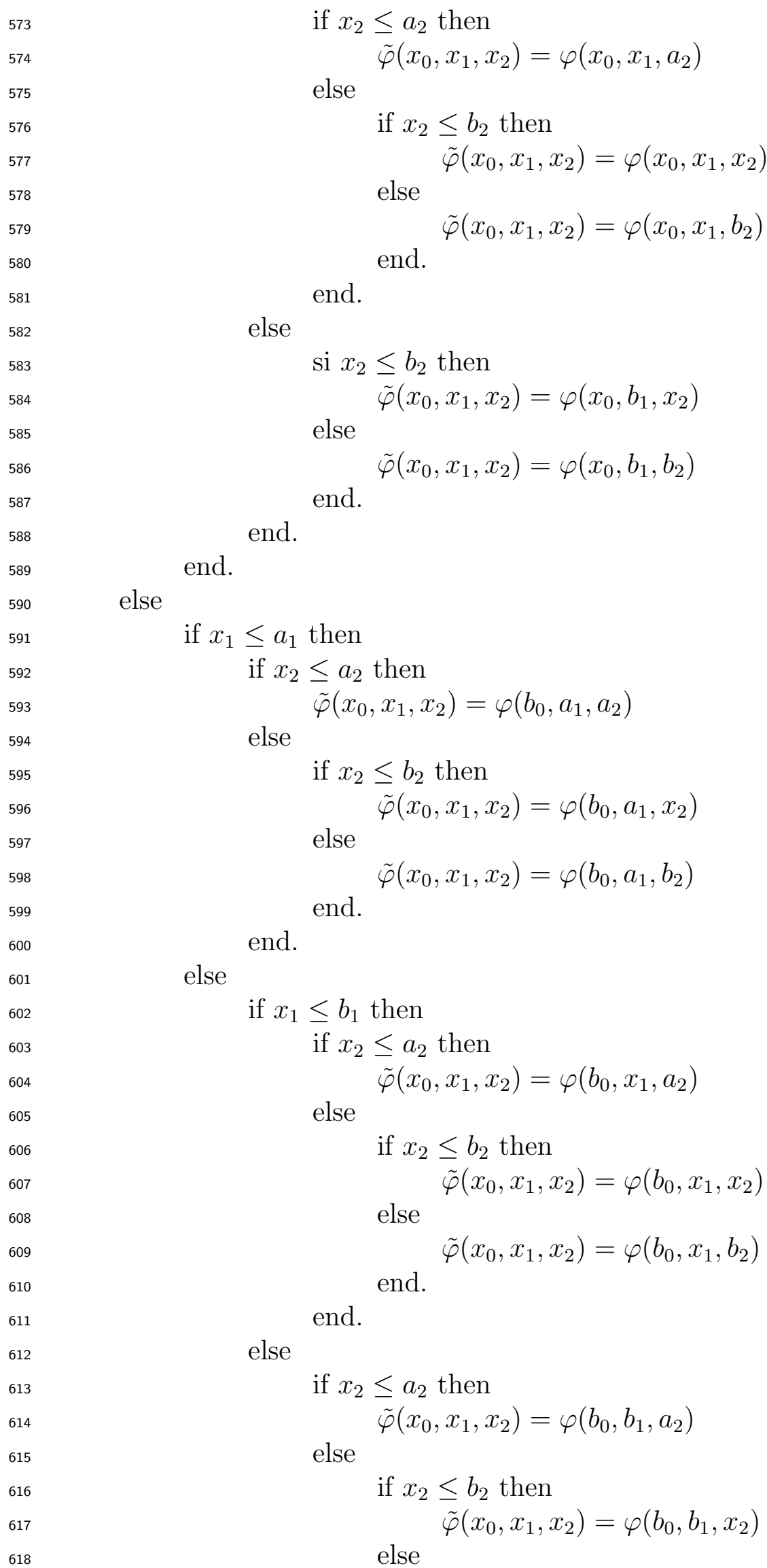


619

620

621

622

623

624

625 end.

end.

$$
\tilde{\varphi}\left(x_{0}, x_{1}, x_{2}\right)=\varphi\left(b_{0}, b_{1}, b_{2}\right)
$$

end.

end.

end.

end. 